\title{
8
}
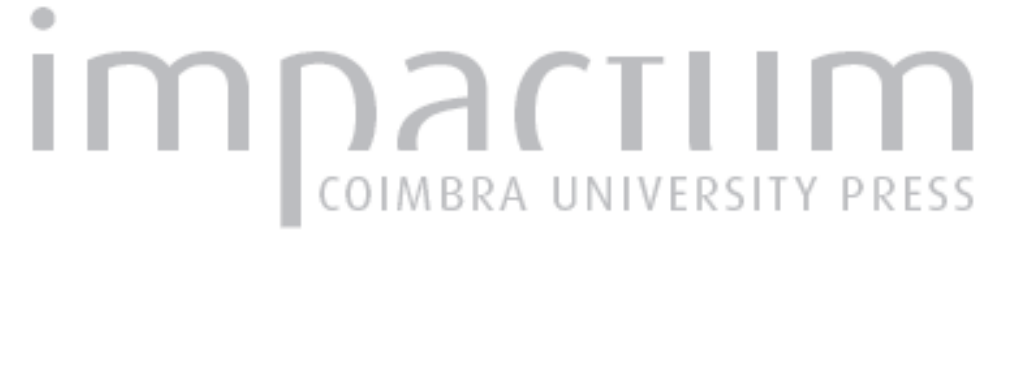

Un pressoir antique creusé dans la roche à proximité de Regina (Casas de Reina,
Badajoz)

Autor(es): $\quad$ Gorges, Jean-Gérard; Rodríguez Martín, F. ${ }^{\circ}$ Germán

Publicado por: Faculdade de Letras da Universidade de Coimbra

URL persistente:

URI:http://hdl.handle.net/10316.2/37704

DOI:

DOI:http://dx.doi.org/10.14195/1647-8657_43_6

Accessed : $\quad$ 26-Apr-2023 08:59:03

A navegação consulta e descarregamento dos títulos inseridos nas Bibliotecas Digitais UC Digitalis, UC Pombalina e UC Impactum, pressupõem a aceitação plena e sem reservas dos Termos e Condições de Uso destas Bibliotecas Digitais, disponíveis em https://digitalis.uc.pt/pt-pt/termos.

Conforme exposto nos referidos Termos e Condições de Uso, o descarregamento de títulos de acesso restrito requer uma licença válida de autorização devendo o utilizador aceder ao(s) documento(s) a partir de um endereço de IP da instituição detentora da supramencionada licença.

Ao utilizador é apenas permitido o descarregamento para uso pessoal, pelo que o emprego do(s) título(s) descarregado(s) para outro fim, designadamente comercial, carece de autorização do respetivo autor ou editor da obra.

Na medida em que todas as obras da UC Digitalis se encontram protegidas pelo Código do Direito de Autor e Direitos Conexos e demais legislação aplicável, toda a cópia, parcial ou total, deste documento, nos casos em que é legalmente admitida, deverá conter ou fazer-se acompanhar por este aviso.

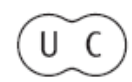


CONIMBRIGA

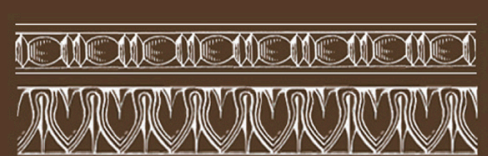

INSTITUTO DE ARQUEOLOGIA

VOLUME XLIII - 2004

FACULDADE DE LETRA

UNIVERSIDADE DE COIMBRA 
JEAN-GÉRARD GorgES

Centre National de la Recherche Scientifique. Université de Toulouse-le-Mirail

UTAH (UMR 5608)

F. ${ }^{\circ}$ Germán Rodríguez Martín

Archéologue. Ancien membre libre de la Casa de Velázquez

\section{UN PRESSOIR ANTIQUE CREUSÉ DANS LA ROCHE À PROXIMITÉ DE REGINA (CASAS DE REINA, BADAJOZ) "Conimbriga” XLIII (2004) p. 149-170}

RÉSUMÉ: Des travaux approfondis de prospections menés sur le territoire de l'antique municipe de Regina, au pied du versant septentrional de la Sierra Morena, ont conduit à la découverte et à l'identification d'un pressoir creusé dans la roche d'un type original. En effet, si la majorité des installations connues pour l'époque romaine sont à mettre en relation avec des exploitations rurales faiblement développées et souvent dans des milieux de tradition indigène, le pressoir de Regina est situé à seulement quelques centaines de mètres du noyau urbain du Ier siècle. Taillé dans le front d'une ancienne carrière de schiste, au milieu d'une zone cadastrée et proche des voies de communication, il apparaît comme un outil destiné à de petits exploitants, au service d'une production limitée et avant tout viticole.

RÉSUMÉ: Los intensos trabajos de prospección en el territorio del antiguo municipio de Regina, situado en las inmediaciones de la vertiente septentrional de Sierra Morena, nos han llevado al descubrimiento e identificación de un original tipo de prensa excavada en la roca. Decimos original, ya que la mayoría de las instalaciones conocidas de época romana, con estas características, se ponen en relación con explotaciones rurales escasamente desarrolladas, y con frecuencia de tradición indígena. La prensa de Regina está situada a menos de un kilómetro del núcleo urbano del siglo I d. C. Tallada en el frontal de una antigua cantera de pizarra, y situada en medio de una zona catastrada próxima a las vías de comunicación, se presenta como una instalación para pequeñas explotaciones, cuya producción es limitada y, sobre todo, vitícola. 
(Página deixada propositadamente em branco) 


\section{UN PRESSOIR ANTIQUE CREUSÉ DANS LA ROCHE À PROXIMITÉ DE REGINA (CASAS DE REINA, BADAJOZ)}

Le municipe flavien de Regina (Casas de Reina, Badajoz), situé au pied des premiers contreforts septentrionaux de la Sierra Morena, appartenait dans l'Antiquité à la province romaine de Bétique. Il dépendait juridiquement du conventus cordubensis. Installée au débouché de voies de passage traversant la Sierra Morena, Regina constitue l'une des étapes citées par l'Itinéraire Antonin pour la voie Astigis-Emerita, laquelle permettait de relier Cordoue (vallée du Guadalquivir) à la capitale de la Lusitanie (vallée du Guadiana).

Connue principalement par les restes de son théâtre antique, cette petite ville en plaine, distincte d'une installation primitive de hauteur occupée aujourd'hui par les ruines de la forteresse almohade de Reina, a été partiellement fouillée dans les années 1980 (Álvarez MARTínEZ, 2001: 45-67 et 1988: 221-229). Son territoire a fait l'objet récemment d'une étude approfondie par les auteurs de cet article, et c'est dans ce cadre qu'a été rencontré et identifié le pressoir que nous présentons maintenant ${ }^{1}$.

1 L'étude du territoire de Regina a été menée entre 2000 et 2003 avec l'appui de la Casa de Velázquez (Madrid); voir les premiers résultats dans: J.-G. GorGES et $\mathrm{F}^{\circ} \mathrm{G}$. RodríGuez MARTín, «De Lusitanie en Bétique: Regina et le réseau routier romain entre Guadiana et Sierra Morena», dans E. Cerrillo, J.-G. Gorges, T. Nogales (éds), Las comunicaciones en Lusitania romana (Cáceres, 2002), Madrid, 2004 (sous presse) et EID., Regina et son territoire (ouvrage en préparation). Nous remercions ici tout particulièrement Jean-Pierre Brun, directeur de recherches au CNRS, pour l'attention avec laquelle il a bien voulu relire ce texte et la pertinence de ses remarques. 


\section{Localisation}

Le pressoir de Regina est situé à environ 800 mètres au sud-ouest du centre de la ville antique et à 600 mètres au nord-est du village actuel de Casas de Reina, non loin du chemin qui conduisait à l'ancienne station de chemin de fer de la RENFE. Il occupe le flanc oriental d'une petite butte (altitude: $645 \mathrm{~m}$ ) connue sous le nom du «Cerro de la Horca». Les coordonnées précises du site sont: $38^{\circ} 12^{\prime} 10,15^{\prime}$ N et 557’43,90”' W (WGS 84).

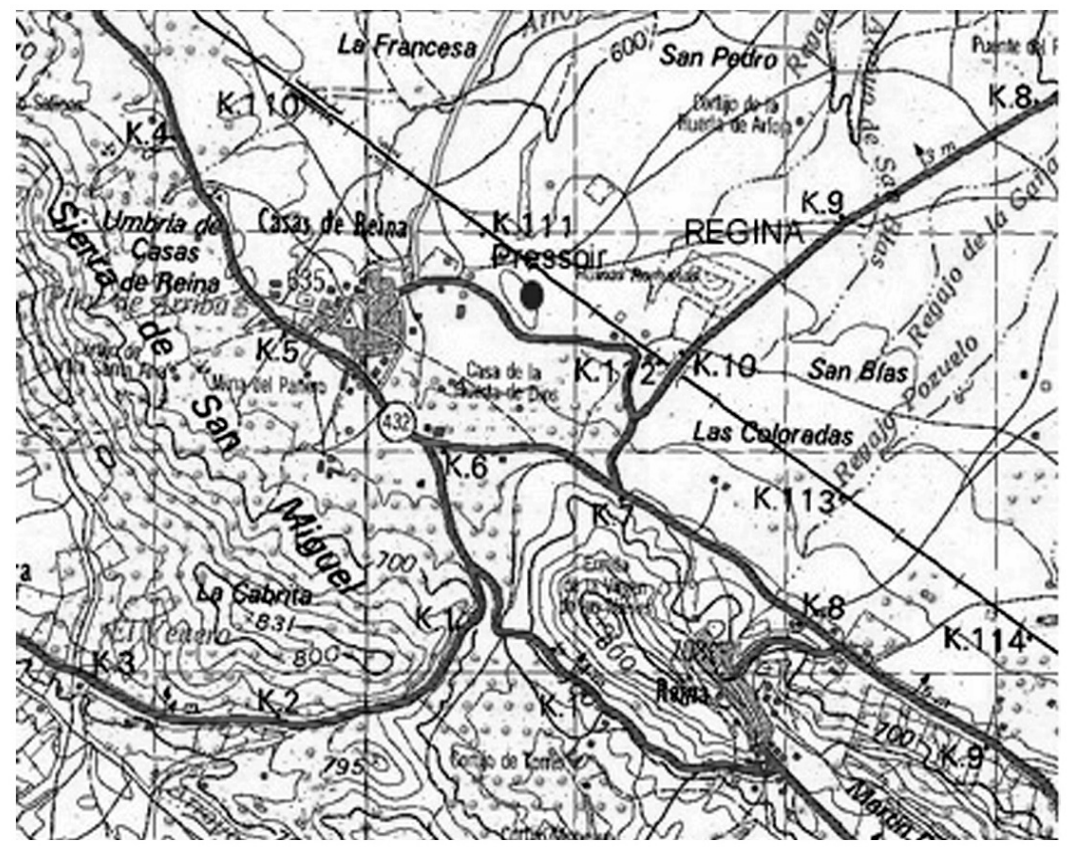

FIG. 1 - Situation du pressoir rupestre de Casas de Reina.

Le gisement occupe un petit affleurement rocheux (schistes), de type batholithe, formant une maigre colline isolée près d'une poche de terres brunes calcaires propices à la culture. L'endroit fut utilisé dans un premier temps comme carrière, notamment pour un approvisionnement partiel en pierre de la ville antique, à l'époque de sa construction. De cette vocation primitive attestent encore de nombreuses traces de coupes, pratiquées dans la roche pour l'extraction des blocs, de même 
que des restes d'encoches destinées à recevoir les coins de bois qui, une fois humidifiés, facilitaient le travail des carriers en gonflant et en provoquant des lignes de faille.

Cette légère élévation présente une perspective différente selon la face par laquelle elle est abordée. Sur les côtés sud-est, sud et ouest, son aspect est plus doux, plus arrondi, avec de faibles affleurements de schistes, alors que sur sa face nord et nord-est on observe dans la roche des coupes plus marquées, résultantes de fronts de taille, ainsi que deux pseudo terrasses. C'est cette paroi, plus abrupte, qui a été mise à profit pour l'aménagement du pressoir.

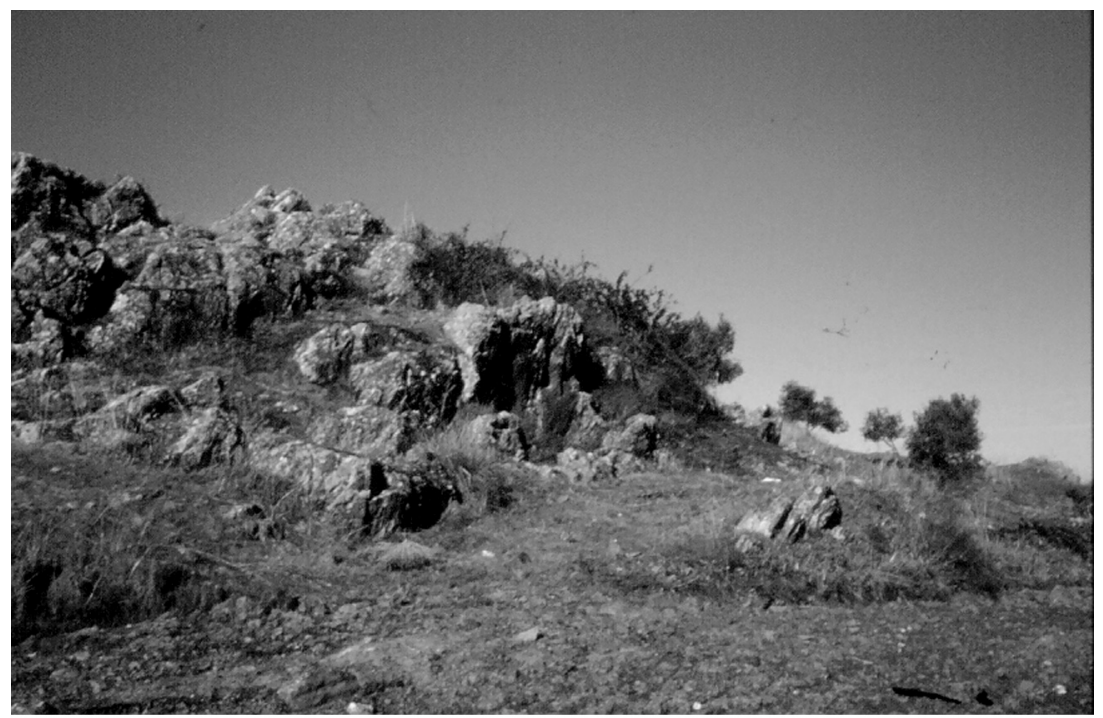

FIG. 2 - Le site du pressoir sur la face nord-est du «Cerro de la Horca».

Les pressoirs creusés dans la roche ne sont pas si fréquents en péninsule Ibérique. Si l'on excepte l'ensemble reconnu² du «Tolmo de Minateda», autour du site supposé de l'antique Ilunum (Hellín, Alba-

2 Cet ensemble réunit une quinzaine de presses en partie taillées dans le rocher, vraisemblablement destinées au pressage des olives: cf. L. ABAD CASAL, S. GuTTIÉRrEZ LLORET et R. SANZ GAMO, «El proyecto de investigación arqueológica 'Tolmo de Minateda' (Hellín, Albacete): nuevas perspectivas en el panorama arqueológico del sureste penínsular», dans Jornadas de Arqueología Albacetense en la Universidad Autónoma de Madrid, Madrid, 1993, p. 147-176. 
cete), c'est dans la province espagnole de Cáceres (GARcía DE FiguEROla Paniagua, 1999) et au Portugal voisin (AlmeidA, 1999 et 1997) que nous rencontrons la majeure partie des exemples de ces installations rupestres, le plus souvent dans des contextes de romanisation ayant conservé un caractère indigène, et sans pouvoir toujours bien établir s'il s'agit d'installations destinées à la production de vin ou d'huile. Toutefois, le pressoir qui nous occupe n'obéit que de loin aux critères qui définissent habituellement les pressoirs creusés dans la roche, encore appelés par les auteurs ibériques «lagar en roca» ou «lagaretas ${ }^{3} » . \mathrm{Ce}-$ pendant, il n'est pas possible non plus de l'inclure dans le lot des pressoirs antiques ordinaires, même si sa conception s'en rapproche fortement.

Les pressoirs en roche connus jusqu'à présent, tant au Portugal que pour le nord de l'Estrémadure espagnole, se caractérisent par leur tendance, pour les plus évolués, à se rapprocher d'un modèle «type» reposant sur l'utilisation d'une surface de foulage et/ou de pressurage (calcatorium), l'existence d'un bassin de réception (lacus), d'un ancrage en roche parfois remplacé par une paire de montants solidement fixés (stipites) de configuration plus ou moins complexe, de jumelles de guidage (arbores), d'un levier ou mouton (prelum) et d'une vis (malus) unie à un contrepoids (ALMEIDA et alii, 1999: 97). Mais il y a bien sûr des variantes, qui peuvent plus ou moins s'éloigner de ce schéma. Par exemple, dans certains cas, le lacus est absent et le moût se recueille alors directement dans des récipients de terre ou des seaux de bois, ou bien encore le calcatorium n'est employé que pour le pressurage. Il y a donc des différences, qui peuvent être notables, même s'il existe un schéma de base.

Si nous considérons un pressoir à vin tel que celui de la villa lusitanienne de Torre Águila (RoDRíguez MARTín, 1999: 263-270; RoDRÍGUEZ MARTín et GORGES, 1999: 403-426), les différences sont minimes par rapport aux éléments utilisés dans cet établissement: aire de foulage (calcatorium), table de pressurage (ara du torcularium), montants d'ancrage (stipites), jumelles de guidage (arbores), bras de presse (prelum),

3 J. GIL MonTES, «La oleicultura romana en el campo Norbense", dans Cuadernos de GRADO. Estudio e Investigación. I. B. 'El Brocense', 3, 1987, p. 78-83, considère que ces installations étaient destinées à la fabrication de l'huile. Elles sont en fait très semblables à celles rencontrées au Portugal, qui sont en général plutôt interprétées comme pressoirs à vin.

Conimbriga, 43 (2004) 149-170 
bassin de fermentation (lacus) et chai (cella vinaria). Il y en a pourtant une essentielle, dans la mesure où le pressoir construit occupe logiquement une position concrète dans l'organisation hiérarchique de la pars rustica de la propriété rurale. D'une manière générale, les structures varieront selon l'importance de la presse, qui cesse avec la villa d'être temporaire pour devenir un élément fixe de l'exploitation. Il s'intègre alors à l'ensemble construit en suivant les recommandations de Palladius (I, 2): orienté au nord, dans un endroit frais et quasi obscur, le pressoir est construit loin des thermes, des fours ou des étables.

De fait, celui de Regina, va se trouver à cheval entre ces deux modèles: le type rupestre d'usage saisonnier et l'installation permanente et protégée de l'exploitation agricole classique de type romain.

\section{Description d'ensemble}

Tel un îlot, la butte formée par l'émergence rocheuse s'individualise par rapport aux terres de culture environnantes. Des deux pseudo terrasses présentes sur le flanc nord-est de ce mamelon, la première, à laquelle on peut accéder par l'est, met à profit une pente naturelle pour atteindre la partie la plus élevée du tertre ainsi formé, après une côte à l'inclinaison moyennement prononcée. Un escalier (?) semble avoir été creusé le long de la paroi la plus haute. A l'extrémité de cette rampe d'accès, en profitant des zones d'extraction des pierres, on a pratiqué à la hauteur du pressoir, dans la bordure de la paroi rocheuse le surplombant, plusieurs logements ${ }^{4}$ dont nous pensons, par la taille et la forme semi-circulaire qu'ils présentent, qu'ils étaient destinés à recevoir et à caler l'extrémité de poutres de bois. Par ailleurs, il semble que l'espace ainsi dégagé au sommet de la rampe, entre paroi haute et paroi basse, bien que de dimensions moyennes, ait pu également être utilisé comme abri, aussi bien quand la carrière était en exploitation que plus tard, quand a fonctionné le pressoir. Le sommet de la butte présente du reste, outre des traces de taille de carriers, quelques débris de tuiles à rebord (tegulae).

4 Il reste les traces de trois de ces logements, mais seul celui qui se trouve en position centrale est bien conservé, offrant une ouverture en demi-cercle bien nette, d'un diamètre d'environ 15 centimètres. 
La seconde terrasse, qui offre la même orientation, est beaucoup plus douce et bénéficie d'un accès facile. Elle était desservie par un chemin ancien la reliant au site de Regina, en traversant la nécropole occidentale de la cité. Occupant la partie inférieure de l'élévation, elle donne l'impression d'avoir été préparée et renforcée par des charges de remblai (cailloutis) pour faciliter la circulation et l'approche des chariots et des personnes jusqu'à l'endroit même où se trouvait le pressoir. Il est toutefois difficile de déterminer s'il s'agit là d'un aménagement antique, en relation avec l'exploitation de la carrière et/ou la mise en œuvre du pressoir, ou bien d'un agencement plus récent.

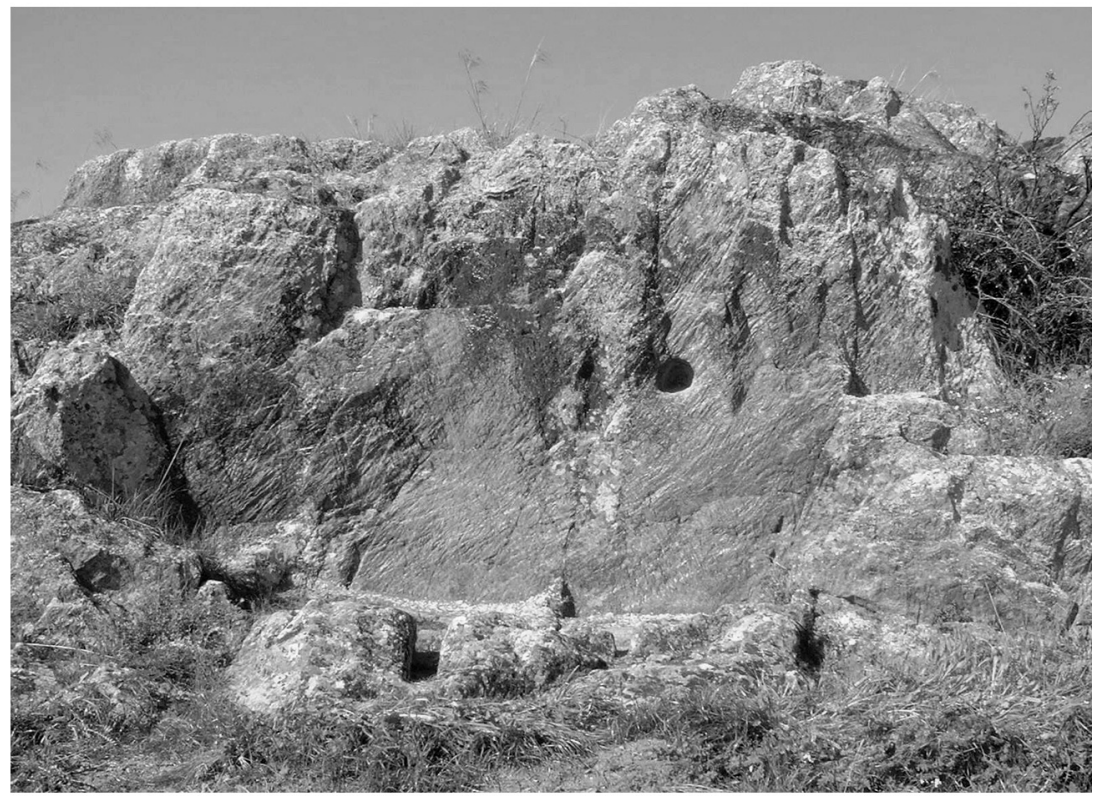

FIG. 3 - Vue de face du pressoir de Regina.

Le pressoir a été réalisé dans la façade nord-est de la carrière antique, en direction de la ville romaine. L'existence d'un front de taille important a certainement été un élément déterminant pour la réalisation in situ d'un tel ouvrage, dans la mesure où il offrait un matériau de qualité déjà en grande partie préparé. Il a fallu toutefois l'aménager pour répondre au nouveau besoin, et pour cela on a mis à profit une paroi, 
haute d'environ trois mètres, qui a de nouveau été travaillée pour obtenir une surface légèrement oblique aussi lisse possible. Bien que dégagée avec soin, les griffes de pics y sont toutefois encore visibles. Sur la partie supérieure, au niveau de la seconde terrasse, on relève les traces de brèches en arcs de cercle déjà signalées. Elles étaient destinées à recevoir des poutres de bois qui devaient servir, vraisemblablement, à supporter une protection de l'ensemble de la zone de travail. Sur cette même paroi, haute de 2,44 m par rapport aux deux cuvettes dont nous parlerons infra, dans la partie centrale correspondant au bassin de droite, une niche a été forée sur une profondeur de $25 \mathrm{~cm}$ pour une ouverture semi circulaire d'un diamètre de 20 à $23 \mathrm{~cm}$. Destiné à recevoir la tête d'un mouton - vraisemblablement constitué ici d'un tronc à l'extrémité plus ou moins oblongue - ce logement est légèrement tronconique, développant un angle interne d'environ $40^{\circ}$, avec une oblique plus prononcée dans sa partie supérieure.

$\mathrm{Au}$ bas de cette paroi, solidaires et creusés directement dans le schiste, deux cuveaux de faible hauteur (de 10 à $20 \mathrm{~cm}$ ) ont été dégagés avec soin. Ils sont séparés par un petit muret également taillé dans la roche - aujourd'hui très détérioré dans sa partie aval - et large d'une vingtaine de centimètres. La première cuve (A), à gauche, mesure 1,30 $\mathrm{m}$ de longueur frontale pour 1,10 $\mathrm{m}$ de largeur. Elle est légèrement plus grande que sa voisine. Très régulière, elle est entièrement dégagée dans la roche et son fond présente une inclinaison approximative de $20^{\circ}$ vers le nord-ouest, où se trouve l'orifice de sortie, constitué par une large saignée $(13 \mathrm{~cm})$ directement pratiquée dans le rebord de pierre. Ce déversoir, qui pouvait facilement recevoir un filtre végétal ou être obturé par une simple lame de bois, donne sur une goulotte droite, relativement profonde, elle aussi creusée dans le roc, laquelle rejoint un autre canal identique arrivant en biais depuis la seconde cuve. L'ensemble aboutit en surplomb au droit d'une petite plate-forme, visiblement destinée à recevoir un récipient. Le second cuveau (B), à droite, mesure 1,05 m en façade pour une largeur identique à $\mathrm{A}$ de $1,10 \mathrm{~m}$. Un peu plus étroit que le premier, il est donc légèrement plus petit, même si son rôle semble avoir été le plus important, comme nous le verrons plus loin. Dégagée au pic, la cuve présente sur son fond la même inclinaison $\left(20^{\circ}\right)$ en direction nord-est. Au point le plus bas, on a pratiqué de la même façon une brèche plus étroite $(8 \mathrm{~cm})$ mais moins profonde que pour le bassin précédent, afin de faciliter l'évacuation des liquides. Cette ouverture est prolongée par un canal en ligne droite sur 
$10 \mathrm{~cm}$ avant de déboucher sur une autre goulotte longitudinale, orientée ouest-est, de largeur similaire. Cette dernière offre la particularité d'avoir été creusée dans la roche de telle manière qu'elle forme une espèce d'arc de cercle vertical, ses extrémités se trouvant à une hauteur supérieure à son point central. Sa pente forme ainsi en cas de débordement de la cuve un déversoir commode vers son point le plus bas à partir duquel le liquide passe alors dans un autre canal qui, après un coude en direction de l'est, rejoint celui issu du premier bassin. Comme le déversoir de A, cette goulotte en arc de cercle vertical pouvait facilement recevoir une lame de bois ou un quelconque système d'obturation destiné à boucher temporairement l'évacuation du bassin B, provoquant alors un débordement au niveau du muret intermédiaire, dont on ne peut exclure qu'il ait pu être doté d'une surverse, comme il arrive fréquemment dans le cas des bassins jumelés.

C'est aussi à ce second bassin que correspond le logement du bras de presse dont nous avons parlé plus haut. Distant en hauteur de 1,20 $\mathrm{m}$ du fond du cuveau, il est placé exactement au centre de celui-ci. Face à lui, à environ 3 mètres de distance de la paroi rocheuse en direction du nord et en contrebas, au niveau d'un léger affleurement de schiste, le sol a été préparé pour permettre la mise en place d'un système de guidage et de soutien du bras du pressoir. Pour cela, on arasé la roche à l'endroit où devaient s'appuyer les montants (stipites) (cf. photo 8). La solidité du roc dispensait ces jumelles de la nécessité d'un bloc d'assise et les deux poteaux verticaux, assemblés à leurs extrémités, reposaient directement sur le sol. Un peu en aval de ce point, le terrain s'abaisse encore de lui-même de plus d'un mètre. C'est là, à 4 mètres de distance du logement du bras de presse, sur une plate-forme bien délimitée, que devait s'effectuer lors du pressurage la manoeuvre du système de contrepoids et le travail sur le levier. 

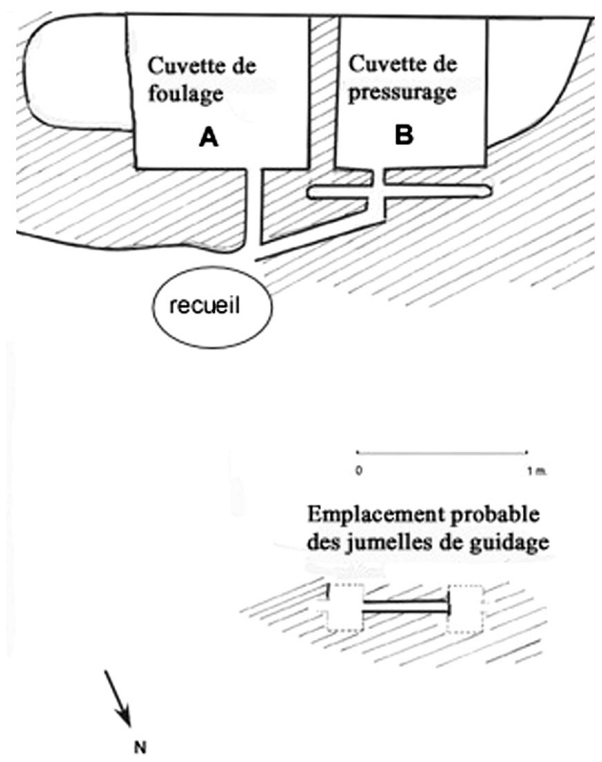

FIG. 4 - Plan schématique au sol (bassins, systèmes d'évacuation, jumelles).

A première vue, ce pressoir pourrait donner l'impression d'être à ciel ouvert. Il convient toutefois d'en rejeter l'idée. En effet, nous avons vu que l'examen du couronnement de la paroi verticale qui surplombe les bassins faisait apparaître des traces de logements - dont un au moins, au centre, est absolument indubitable - destinés à l'encastrement et au maintien de madriers de bois. Ces poutres horizontales, qui devaient être combinées à un jeu de poteaux verticaux et de cordes, Iformaient la charpente d'une couverture abritant l'ensemble de la zone de travail. Cet aspect particulier de la protection des pressoirs rupestres, qui n'est pas sans avoir suscité de sérieux doutes (BROCHADO et alii, 1999: 102), trouve ici une réponse. Il n'est pas interdit de supposer, dans le cas qui nous occupe, que cette protection pouvait être assurée par une toiture légère et des panneaux latéraux formés de branchages et de végétaux entrelacés (genêts, joncs, chaumes...), sur le modèle de ceux qui sont encore utilisés dans la région pour les abris destinés au bétail ou les cabanes de bergers (chozos). La mission d'une telle couverture ne se limitait sans doute pas protéger cycliquement la presse et ses opérateurs 
des inclémences de la météorologie - qu'il s'agisse du soleil, du vent ou de la pluie - mais elle devait également se prolonger dans le temps en assurant une protection plus durable à une installation certes a priori robuste, mais qu'il n'en fallait pas moins préserver, indépendamment du fait qu'on ait pu démonter l'ensemble des parties «mobiles» la saison finie, ce dont on peut cependant douter.

\section{Tous les éléments d'un pressoir à vin}

Quelle structure globale présentait donc ce complexe de pressurage et pour quel produit ? Si l'on s'en reporte au plan d'ensemble (Fig. 4), on observera que deux parties se détachent nettement: l'une correspond à la cuve orientale $\mathrm{A}$, l'autre au bassin ouest $\mathrm{B}$. La première partie est la plus simple, puisqu'elle correspond à la seule cuvette, la plus grande. Ainsi que nous l'avons vu, elle est entièrement creusée dans la roche mère, et son fond incliné conduit vers une ouverture qui donne sur un canal de sortie relativement profond. La seconde cuvette $\mathrm{B}$, bien qu'un peu plus petite, présente un schéma plus compliqué: elle est en effet surmontée en son centre, sur sa paroi verticale, à une hauteur de quatre pieds romains $(1,20 \mathrm{~m})$, d'une niche assez profonde, légèrement tronconique, dont l'axe se retrouve en ligne avec les traces d'ancrage d'arbres intermédiaires relevés à trois mètres de là (soit environ dix pieds). Il n'y a pas de doute sur le sens de cette cavité, qui correspond au logement de la tête du bras de presse, dont on voit bien qu'il devait agir comme un levier puisque ce logement est précisément creusé en oblique, notamment dans sa partie supérieure. Compte tenu de la grosseur du bras - entre 15 et $20 \mathrm{~cm}$ - l'angle de débattement possible avoisinait les $10^{\circ}$ à $15^{\circ}$, ce qui donnait une amplitude de mouvement d'environ $15 \mathrm{~cm}$ au niveau du plateau de presse, au centre du cuveau B. Le diamètre limité du prelum, son faible débattement et sa longueur qui ne devait pas excéder de beaucoup les quatre mètres (emplacement présumé des contrepoids), tout cela plaide pour une installation de puissance moyenne, plutôt faible pour l'huile mais bien suffisante pour le vin, d'autant que le bassin A, qui ne présente aucune sophistication particulière mis à part son système d'écoulement direct, ne peut correspondre qu'à un bassin de foulage (calcatorium). En effet, bien que de dimensions réduites et de faible hauteur (1,30 m x 1,10 m x 0,20 m), ce fouloir ne doit pas tromper: une fois muni d'un cadre de planches des- 
tiné à le rehausser et à recevoir les grappes, il devait être rempli sur une hauteur de 25 à $30 \mathrm{~cm}$ au moins et deux hommes pouvaient facilement y trouver place, écrasant les grains en cadence tout en se maintenant, pour ne pas glisser, à des cordes qui pouvaient être fixées à l'un des madriers de la charpente. C'est là que se menait la première phase du processus de vinification, avec l'obtention d'un premier moût (BLANCO et RougGeAu, 1999: 124), dont l'importance était d'ailleurs capitale. On sait en effet que le produit du foulage n'a pas la même composition chimique que le jus issu du pressurage (BRUN, 1997: 67). Les Anciens recommandaient d'ailleurs vivement de ne pas mélanger de manière inconsidérée les différents moûts ${ }^{5}$. La seconde phase se déroulait en B, qui correspond au bassin de pressurage, là où travaillait le plateau de presse. Après avoir été foulé en A, le marc restant (peau, pépins, jus, résidus divers...) était donc rapidement transvasé en $\mathrm{B}$, pour y être pressé à proprement parler. Selon Pline, ce dernier processus pouvait se reproduire plusieurs fois, donnant un vin de moindre qualité à mesure que se succédaient les opérations. On sait du reste que les derniers pressurages, une fois la masse épuisée, faisaient l'objet d'un ajout d'eau pour obtenir un «vin de marc» (vino acinaticium) qui ne durait pas trois mois, et que l'on donnait aux esclaves'.

Pour le premier processus, étant donnée la faible hauteur qu'offre le cuveau $\mathrm{A}$ - dont l'un des angles est par ailleurs très abîmé - on devait vraisemblablement y placer des montants en bois de manière à former une caisse haute de plusieurs dizaines de centimètres, comme nous l'avons déjà évoqué. Dans ces conditions, le liquide produit par le foulage pouvait être assez abondant, ce qui pourrait expliquer la taille relativement importante de l'orifice de sortie, où l'on devait aussi pouvoir placer des filtres végétaux destinés à récupérer une partie des pépins et des peaux. De la même façon, pour le pressurage, on pouvait placer en B une cage en planches pour contenir le moût, mais cette fois d'une hauteur plus importante (sans doute 40 à $50 \mathrm{~cm}$, peut-être plus) compte tenu du niveau auquel travaillait l'arbre. Toutefois, des exemples traditionnels montrent que l'on pouvait aussi utiliser une grosse corde enroulée en cercle pour maintenir le marc, voire même le presser directe-

5 Pour Varron (De Re Rustica, I, 54, 3), on peut mélanger le vin de goutte et le vin de première pressée, mais on doit séparer le vin de seconde presse, dit «vin de rognures», parce qu'il a «un goût de fer».

6 C'est ce qu'on appelle la «piquette», ou lora. 
ment en tas, en coupant ensuite ce qui dépassait pour le presser à nouveau (vinum circumcisum). Du fait de cette situation de l'ancrage du prelum à une hauteur de 1,20 m par rapport au fond du bassin, la force du levier s'appliquait nécessairement sur le plateau de pressage par l'intermédiaire d'un jeu de cales et de traverses dont le nombre pouvait être ajusté en fonction de la hauteur réelle de la cage ou de la masse accumulée. Au fur et à mesure des différentes étapes du pressurage, le jus pouvait s'écouler indistinctement par l'évacuation du cuveau B ou du cuveau A en passant par-dessus le muret qui les séparait, puisque dans tous les cas les deux bassins étaient juxtaposés et que leur inclinaison favorisait un écoulement dans le même sens. Rappelons par ailleurs l'existence devant la cuvette B d'une assez profonde rigole longitudinale et arquée, avec des extrémités plus élevées et une inclinaison vers un canal central d'évacuation, dont la destination était précisément de recueillir le moût pouvant déborder par la façade de ce même bassin.

Le jus obtenu ici, à la différence de ce qui se passait dans un pressoir traditionnel, ou même avec certains pressoirs en roche, ne venait pas s'accumuler dans une cuve appropriée (lacus) où on le laissait le temps nécessaire pour que se produise la fermentation indispensable à la vinification, la fameuse «ébullition tumultueuse». Dans le cas présent, il ne pouvait être recueilli qu'en récipients externes qui étaient ensuite transportés dans la cella vinaria de la demeure de son propriétaire, ou tout au moins dans l'endroit qui tenait lieu de chai, et c'est là que s'effectuait la suite naturelle du processus. Le transport était facilité par l'accès aisé des carrioles à la zone du pressoir, et il est certain que le chargement ou le déchargement des jarres ou des divers récipients, comme celui des raisins à traiter, ne devait pas poser de problème particulier. Par ailleurs, au niveau même des cuvettes, les parois latérales ont été largement taillées en biais, moins sans doute pour servir de possible déversoir d'alimentation du fouloir en A - par exemple - que pour faciliter d'une façon générale les conditions de travail des servants de l'installation en leur donnant davantage d'espace pour les diverses manipulations.

Ainsi, quelle que soit l'originalité de ce pressoir et sa situation, il n'existe pas de véritables différences avec ce qui se serait passé en utilisant le pressoir traditionnel d'une villa, ni dans le processus de vinification, ni dans l'outillage essentiel et les méthodes employées. Tout au plus, dans le cas du pressoir de Regina - et mise à part encore une fois 
l'absence de lacus - l'originalité résiderait-elle dans le système d'ancrage de la tête de presse. Celle-ci s'appuie d'ordinaire sur des montants ou des jumelles spécifiques (arbores), souvent pris dans le gros œuvre, et qui bien sûr n'ont pas lieu d'être ici puisque la tête du prelum vient directement se loger dans la roche, dont la résistance à l'effort est bien évidemment plus que suffisante. Le dernier tiers du bras reposait sur des arbres intermédiaires (stipites), placés non loin de l'extrémité de la poutre, pour former une zone de guidage et de régulation du pressurage, comme on en retrouve sur toutes les presses qui ne sont pas de simples leviers à bras. Leur emplacement s'apprécie au sol, à 3 mètres environ $\mathrm{du}$ point d'ancrage en paroi (cf. photo 8); ces montants, hauts d'au moins 2 mètres du fait de la configuration du site, devaient pouvoir supporter à cette distance un jeu vertical du mouton d'environ 70 à $80 \mathrm{~cm}$; ils pouvaient également porter en partie haute une poulie destinée à le relever plus facilement, à l'aide de cordages ou de courroies de cuir. Pour assurer le maintien du prelum en position semi horizontale et contrôler le débattement du bras de presse, il est probable qu'ils aient été dotés de fentes verticales ménagées dans les flancs et munies d'un système de cales amovibles pour en réguler le mouvement. Enfin, il est à peu près certain, par la topographie et l'agencement visible du terrain, que l'ensemble se terminait par un système de contrepoids pour tirer le levier vers le bas. On en retrouve au sol l'emplacement privilégié en contrebas, à une distance d'environ 4 mètres de la paroi d'ancrage.

Il est difficile de savoir quel modèle était utilisé - à vis, à balancier ou à treuil - car il ne reste en place aucun vestige de ce type de matériel, hormis l'espace nécessaire pour l'accueillir. Toutefois, il est possible d'esquisser quelques réponses: la sophistication de l'ensemble, en effet, paraît devoir exclure l'archaïque système à balancier et le diamètre relativement faible de l'arbre - entre 20 et $23 \mathrm{~cm}$ d'après les dimensions de la niche qui accueillait la tête du prelum - rend problématique du point de vue de la résistance à l'effort le passage d'une vis dans un tel bras, sauf à imaginer un système complexe d'attache. Nous aurions donc tendance à privilégier soit un système léger de contrepoids à vis 7 , soit un système plus classique de contrepoids à treuil.

7 En utilisant par exemple, comme pour beaucoup d'appareils de faible puissance (le vin ne nécessite pas une très grande puissance pour le pressurage), un tronc fourchu: on place alors dans la fourche un écrou destiné à recevoir la vis, elle-même reliée à un contrepoids de pierre adapté à la grosseur du levier. 


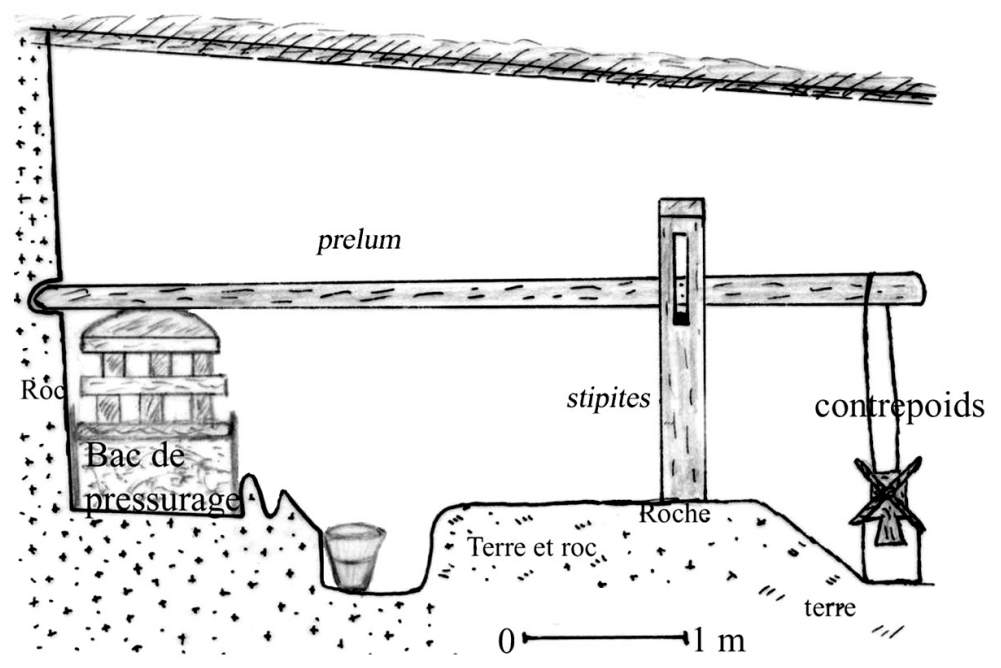

FIG. 5 - Essai de restitution schématique du pressoir de Regina.

La dimension des cuvettes semble indiquer que la production ne devait pas être très considérable, mais plutôt destinée à satisfaire une consommation privée. Néanmoins, il n'a pas été possible de mettre clairement ce site en relation avec une installation rurale de proximité. Les établissements les plus proches - villas ou sites secondaires - sont tous distants d'environ 400 à 500 mètres, au sud du point considéré, et correspondent à une concentration de petites exploitations autour de bonnes terres facilement irrigables (lieu-dit «Huerta de Dios»). Quatre sites au moins ont été repérés, séparés entre eux de 150 à $250 \mathrm{~m}$. Ils correspondent à des alignements du cadastre antique que nous avons pu relever autour de Regina. ${ }^{8}$ Aucun autre site n'a pu être localisé au nord, à l'ouest ou à l'est du Cerro de la Horca, à une distance inférieure à 1.000 ou $1.500 \mathrm{~m}$. Il est vrai aussi que les terres sont là moins profondes et que le noyau urbain ne se trouve qu'à $800 \mathrm{~m}$ à peine en ligne droite du pressoir. Ce dernier était relié à la ville par une route passant à son pied, laquelle a naturellement pu drainer un certain nombre de propriétaires des parcelles environnantes.

8 Voir les détails dans la bibliographie citée en note 1. 
Cette impossibilité de mettre en relation le pressoir rupestre de $R e$ gina avec un établissement précis, ses connexions avec différents groupes d'utilisateurs potentiels, sa situation dans une carrière, ou une ancienne carrière, qui comme telle pouvait encore relever de l'ager publicus ${ }^{9}$, tout cela nous conduit à émettre l'hypothèse prudente d'un éventuel instrument à usage collectif. On imagine mal, en effet, une exploitation précise faisant construire pour son seul usage un pressoir placé à près d'un demi kilomètre ou plus de son bâtiment principal, le tout pour une production vraisemblablement modeste mais qu'il fallait encore transporter ensuite jusqu'à son lieu normal de stockage. Quoiqu'il en soit, propriété publique ou propriété privée, c'est précisément cette possibilité d'utilisation collective qui, à nos yeux, pourrait expliquer l'absence de tout bassin (lacus) destiné à la fermentation du vin, alors que la place ne manquait pas et que la roche s'y serait parfaitement prêtée. On pourrait donc imaginer que certains petits propriétaires de parcelles proches du municipe - dont certains pouvaient résider dans la ville basse comme dans la ville haute plus ancienne - aient eu recours à un tel outil. Si tel était bien le cas, il faudrait alors penser à une utilisation par un assez grand nombre de familles tout au long de l'époque des vendanges, chacune emportant le produit de sa vigne, ce qui bien sûr rend difficile l'appréciation globale de la quantité de vin obtenue durant chaque campagne au moyen d'une installation qui apparaît, de toutes façons, loin d'être rudimentaire.

Un tel système serait en adéquation avec les résultats des prospections menées, qui font apparaître un nombre relativement faible d'habitats dispersés à proximité immédiate de la cité, et qui privilégient les constructions modestes aux dépends des villae traditionnelles, notamment dans la zone centuriée que nous avons pu mettre en évidence ${ }^{10}$. Il est clair par ailleurs qu'on ne saurait parler de grande production, sinon de petites productions, à usage plutôt familial, même si l'on ne peut exclure l'existence d'un modeste commerce local. La culture de la vigne - par ailleurs coûteuse, délicate et nécessitant une surveillance

9 On sait qu'à Mérida (Augusta Emerita), les grandes carrières relevaient du domaine public: il en était ainsi, par exemple, pour les carrières de granit du pantano de Proserpine.

10 Les terres agricoles proches de Regina ont bénéficié par deux fois d'un système de cadastration antique qui a laissé sa marque dans le paysage, l'un s'appuyant sur la ville haute (oppidum), l'autre sur la fondation nouvelle en plaine, les deux noyaux urbains ayant coexisté en grande partie sous le Haut-Empire. 
réelle - devait s'effectuer tout autour de Regina et sans doute, comme cela se passait dans les campagnes cordouanes, en association avec d'autres types de cultures - figuiers, oliviers... - de la même manière que pour les arbres fruitiers.

\section{Une improbable utilisation pour la production d'huile}

Le pressoir en roche de Regina pouvait-il aussi servir pour la production d'huile? On pourrait évidemment se poser la question. D'abord parce qu'une bonne partie des infrastructures en place et des matériels induits pouvaient tout aussi bien servir à la production d'huile qu'à celle du vin. Ensuite parce que le calendrier des vendanges et celui de la récolte des olives pour la production d'huile répondent à des temps différents: de la fin août à la mi-septembre au moins pour la récolte des raisins et la production du vin; l'automne et le début de l'hiver pour la cueillette des olives et la production d'huile.

Il est peu probable, cependant, qu'il en ait été ainsi, même si l'existence des deux cuveaux jumelés et leur séparation par un petit muret qui aurait pu être équipé d'une surverse pourraient correspondre à une caractéristique fréquente des pressoirs à huile. C'est en fait le seul véritable argument qui serait à mettre au crédit de cette hypothèse, mais il se trouve que l'on connaît également des dispositifs similaires pour le vin afin de décanter les rafles, comme à Pompéï, par exemple. Nous n'avons par ailleurs aucune trace de meule ou de moulin à proximité: indispensables au broyage ou a la trituration préalable des olives, ces outils auraient pu, en partie, être réalisés dans la roche, comme il arrive fréquemment pour les huileries rupestres, ce qui n'est pas le cas ici. Enfin, le pressurage des olives nécessite ordinairement de très fortes pressions, et donc des installations puissantes même si elles peuvent être parfois sommaires (presses à vis ou à fort levier), ce qui ne semble pas véritablement correspondre au profil de notre pressoir. Quant au double usage un instant suggéré plus haut, il faut bien reconnaître que, outre qu'il suppose un nettoyage extrêmement minutieux des cuveaux et du matériel, il se présente toujours comme une exception ${ }^{11}$. Pour ces

11 On le voit bien dans les villae traditionnelles, où la pars rustica rassemble habituellement les deux types d'installations, donnant à l'une ou à l'autre plus ou moins d'importance selon les époques et le produit privilégié.

Conimbriga, 43 (2004) 149-170 
raisons, il semble raisonnable de penser que le pressoir de Regina, qui apparaît pleinement fonctionnel et parfaitement adapté à l'élaboration du vin, n'ait servi qu'à l'élaboration de ce seul produit.

\section{Datation}

Bien que rupestre et creusé en roche, et sans doute essentiellement destiné à la vinification, le pressoir de Regina s'apparente clairement au type A3 de la classification des pressoirs à levier établie par J.-P. Brun dans son étude sur les huileries du département du Var (BRUN, 1986: 86). Ancré dans une paroi, le bras de presse est guidé par des jumelles et manoeuvré par un treuil fixé sur un contrepoids. Si un tel modèle trouve sa diffusion dans le monde antique dès le IIe s. avant J.-C., il est évident que notre pressoir ne remonte pas si haut dans le temps. Le lieu de son installation, toutefois, peut nous fournir des éléments chronologiques relativement plausibles. Le Cerro de la Horca est situé, on l'a vu, à moins de 800 mètres du centre de la cité romaine. Son utilisation partielle comme carrière dès l'époque antique ne fait aucun doute. Même si son exploitation a commencé antérieurement à l'édification de la ville, il est probable que celle-ci a surtout été utilisée au moment où la cité se développait. Or la Regina en plaine, promue municipe vraisemblablement sous les Flaviens, n'apparaît que sous les derniers julioclaudiens, au plus tôt sous le règne de Claude, si l'on se fie à la céramique retrouvée lors des fouilles. Son développement, demeuré très faible en dépit de la construction d'un théâtre de 800 places, ne semble pas s'être poursuivi au-delà de la fin du Ier s. ou du début du IIe s. ap. J.C. Par ailleurs, l'excellent travail de la taille de la paroi et des bassins de l'installation portent la marque d'une facture du Haut-Empire. C'est donc vraisemblablement à partir de cette époque - soit entre la fin du Ier s. et la fin du II ${ }^{e}$ s. ou les premières années du IIIe s. ap. J.-C. - que la carrière a cessé d'être exploitée et qu'a pu se produire sa transformation partielle en pressoir dont l'utilisation, en revanche, a pu perdurer fort longtemps, de façon plus ou moins intermittente. La villa de Torre Águila nous offre un bon exemple de la longue vie (ou survie?) dont ont pu bénéficier certaines presses, puisqu'au delà des abandons successifs, y compris quand la villa n'était plus que ruine, le pressoir à vin a continué de fonctionner, et cela jusqu'à l'époque arabe incluse (RODRÍGUEZ MARTín et GoRGES, 1999: 425-426)! 


\section{Conclusions}

Il paraît clair que le pressoir de Regina représente à sa façon un unicum et n'est en définitive en consonance ni avec les installations rupestres connues pour la province de Cáceres, ni avec celles du Portugal. Seul le pressoir de «Sabões da Mina», à Bensafrin, en Algarve, présente un schéma plus ou moins similaire (RocHA, 1896: 65-79): calcatorium taillé dans la roche, prelum encastré dans une paroi, bien que le système d'ancrage du mouton soit différent et qu'en l'espèce il y ait bien un lacus creusé dans la roche, ce qui n'est pas le cas à Regina. Il reste que ces deux exemples représentent un modèle déjà élaboré qui s'éloigne grandement de la majorité des pressoirs rupestres répertoriés pour la péninsule Ibérique. Toutefois, compte tenu de sa situation, de l'ensemble des éléments qu'il présente ou induit et de sa structure sous abri, le pressoir de Regina est celui qui se rapproche le plus des pressoirs traditionnels propres aux établissements ruraux d'époque romaine. Par ses capacités, ses dimensions, il semble représenter en moyenne un potentiel égal à environ 50\% d'un pressoir ordinaire de villa, ce qui le rend particulièrement apte à traiter des séries de volumes relativement réduits. N'offrant aucune capacité de stockage propre, intermédiaire entre un instrument de production classique et de production strictement familiale, il semble bien correspondre à ce qui était apparemment sa vocation première: constituer un outil simple, pratique et performant, au service de petits propriétaires du terroir d'une modeste cité romaine du piémont de la Sierra Morena, écartelée entre Bétique et Lusitanie.

\section{BIBLIOGRAPHIE}

Blanco, José Luís; Rouggeau, Michelle (1999): “Técnicas para la elaboración del vino», El vino en la antigüedad romana (Jérez, 1996), Madrid, 263-270.

Brun, Jean-Pierre (2003): Le vin et l' huile dans la Méditerranée antique: Viticulture, oléiculture et procédés de fabrication, Paris.

BRUN, Jean-Pierre (1997): "Production de l'huile et du vin en Lusitanie romaine", Conimbriga, 36, 45-72.

BRUN, Jean-Pierre (1986): L'oléiculture antique en Provence: les huileries du département du Var, Paris.

CARdozo, Mário (1946): «Lagares dos mouros», Actas y Memorias de la Sociedad Española de Antropología, Etnología y Prehistoria, 21, 1:4, 134sq. 
Carvalho, António (1994): «Lagares de vinho e seus componentes nas villae romanas do território português», O vinho, a História e a cultura popular (Actas do Congresso $O$ Vinho na Festa da Cultura), Lisbonne, 39-72.

Cerrillo, Enrique (1999): «La producción doméstica del vino en la antigüedad romana: entre las recomendaciones de los agrónomos y las evidencias arqueológicas”, El vino en la Antigüedad romana (Jérez, 1996), Madrid, 63-78.

FERNÁNDEZ CASTRO, María Carmen (1983): «Fábricas de aceite en el campo hispanoromano», El aceite en la Antigüedad, (Actas del II Congreso internacional sobre producción y comercio del aceite en la Antigüedad), Madrid, 569-599.

García de Figuerola Paniagua, Miguel (1999): Arqueología romana y altomedieval de la Sierra de Gata (El valle de Valverde, Provincia de Cáceres), Cáceres.

GonZÁlez BlanCO, António (1993): «Pressoirs à huile d'époque romaine dans la péninsule Ibérique», La production du vin et de l'huile en Méditerranée (Supplément au BCH, XXVI), Paris, 397-411.

JúnIOR, José Ribeiro dos Santos (1989): "Um lagar luso-romano do vinho junto do santuário de Panoias", Revista de Guimarães, XCIX, 411-416.

LAPORTE, Jean-Pierre (1985): «Fermes, huileries et pressoirs de Grande Kabylie», Bulletin Archéologique du Comité des Travaux Historiques et Scientifiques, NS 19, B, (II ${ }^{\mathrm{e}}$ colloque international sur l'Histoire et Archéologie de l'Afrique du Nord [Grenoble, 5-9 avril 1983]), 127-146.

LeVeau, Philippe; Sillières, Pierre; Vallat, Jean-Pierre (1993): Campagnes de la Méditerranée romaine, Paris.

LóPEZ, Alberto; RicARdo, Batista; ZuCCHITELlo, Mario (1985): «La producción vitivinícola de la Tarraconense. Algunos ejemplos sintomáticos», El vi a l'antiguitat. Economia, producció i comerç al Mediterrani occidental, Badalona, 319-325 .

LOUREIRO, Virgílio (1994): «As lagaretas escavadas na rocha: uma perspectiva tecnológica», $O$ vinho, a História e a cultura popular (Actas do Congresso O Vinho na Festa da Cultura), Lisbonne, 27-39.

MezQuíriz de Catalán, Maria Angeles (1995-1996): «La producción de vino en época romana a través de los hallazgos en territorio navarro», Trabajos de Arqueología Navarra, 12, 63-89.

Rocha, António dos Santos (1896): "Notícia de algumas estações romanas e árabes do Algarve”, O Arqueólogo Português, II, 65-79.

Rodríguez Martín, Fº Germán; Gorges, Jean-Gérard (1999): “Prensas de aceite y de vino en una villa romana de la cuenca media del Guadiana (Torre Águila, Barbaño, Badajoz)", Économie et territoire en Lusitanie romaine, Madrid, 403-426.

RodríGUEZ MARTín, F Germán (1999): "Vestigios de la explotación del vino en la villa romana de Torre Águila (Montijo, Badajoz): el lagar”, El vino en la antigüedad romana (Jérez, 1996), Madrid, 263-270.

RodríGuEz MARTín, F Germán (1990): “Algunas consideraciones sobre la producción de aceite en las Vegas del Guadiana (Talavera-Barbaño), durante los primeros siglos del Imperio (I-III d.C.)", XII Jornadas de viticultura y etnología de tierra de Barros, Almendralejo, 273-281. 
RodríGuez MARTín, $\mathrm{F}^{\circ}$ Germán (1988): "Prensas y lagares de la villa romana de Torre Águila. Barbaño-Montijo (Badajoz) ", X Jornadas de viticultura y etnología de tierra de Barros, Almendralejo, 223-242.

TCHERNIA, André; BRUn, Jean-Pierre (1999): Le vin romain antique, Paris.

TORRe RodrígUeZ, José Ignacio de la (2000): "Aportaciones al estudio de la viticultura en la região demarcada do Porto durante la antigüedad tardía”, Hispania Antiqua, XXIV, 299-317. 


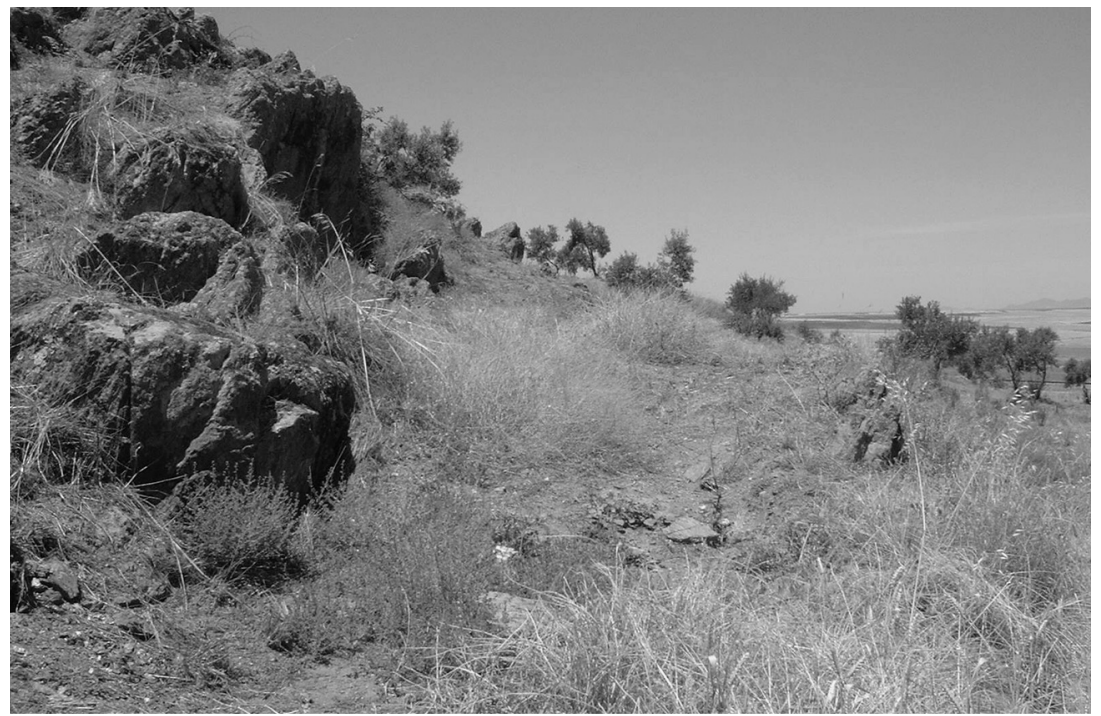

Рното 1. - Vue de l'accès au pressoir: on notera l'abord aisé facilité par une terrasse en pente douce et l'échelonnement du terrain face à l'ouvrage qui permettait l'étagement des parties annexes de l'installation (jumelles et contrepoids).

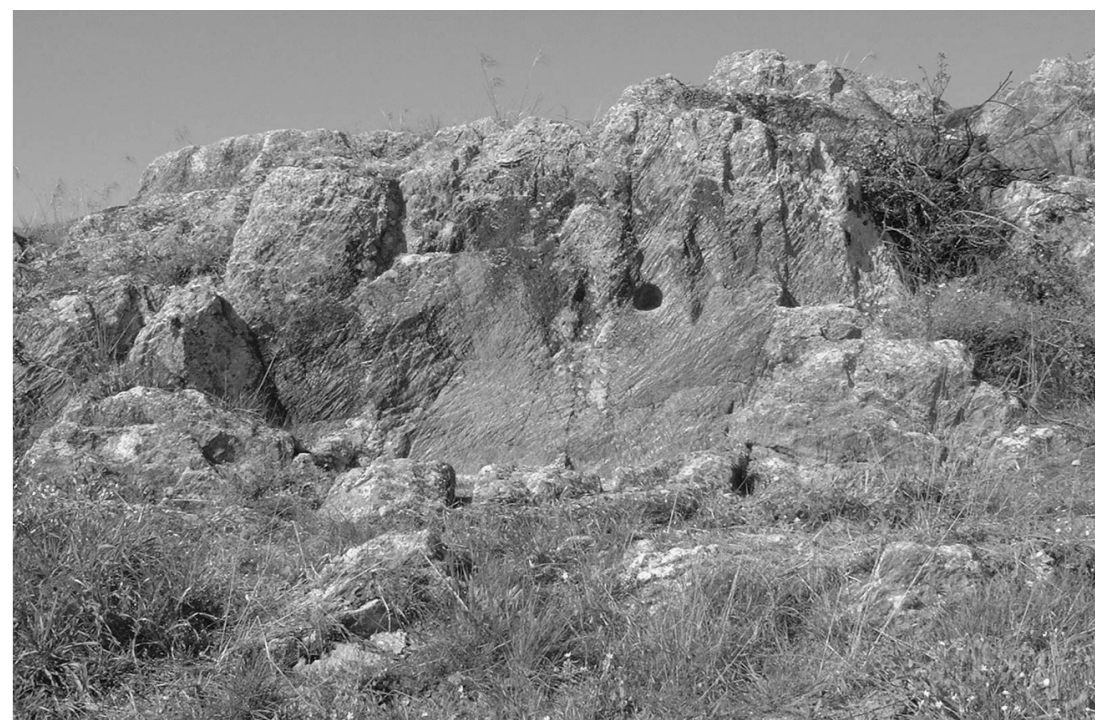

Рното 2. - Le pressoir vu de face. La paroi verticale dégagée au pic dans la roche est plus large que celle nécessitée par les deux bassins, afin de faciliter une accessibilité latérale. La niche d'ancrage du levier est bien visible à droite, au centre du bassin de pressurage, de même que, à gauche, le canal d'évacuation de la cuvette de foulage. Sur le couronnement, auquel on accédait facilement par une seconde terrasse, des encoches pouvaient recevoir la tête d'une poutraison destinée à protéger la surface de travail. 


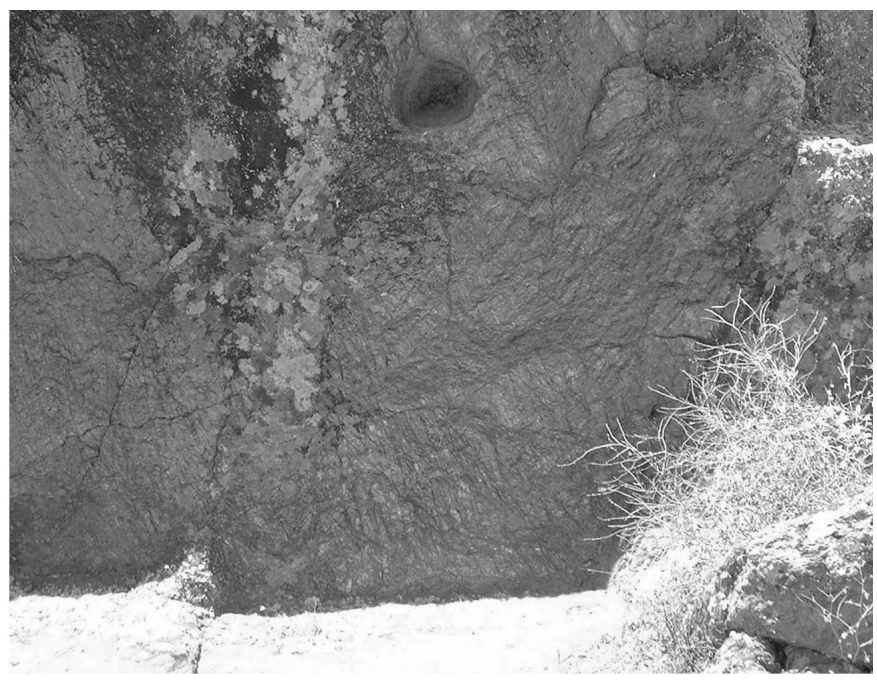

Рното 3. - Détail de la partie correspondant au bassin de pressurage. On observera l'aspect tronconique de la niche d'ancrage de la tête du bras de presse, ainsi que son ouverture angulaire vers le haut, la position horizontale devant marquer la fin du débattement du levier. En bas, à gauche, on aperçoit les vestiges du muret de séparation entre les deux cuvettes, dont l'inclinaison est visible.

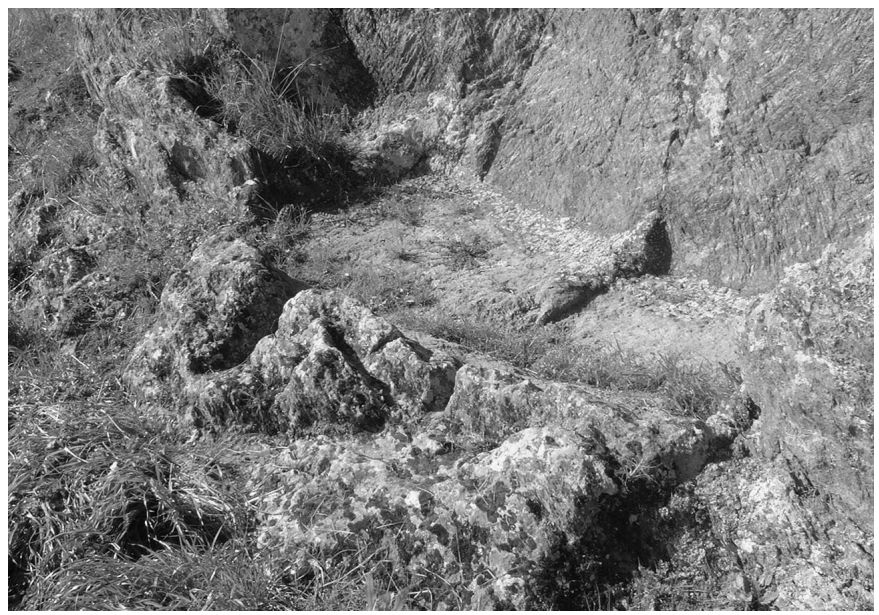

Рното 4. - Vue de trois quarts des deux cuvettes et de leur système d'écoulement. On remarquera que tout a été fait pour dégager latéralement les bassins (notamment celui du calcatorium) et les rendre plus «manoeuvrables»: la paroi «verticale» forme elle même un plan légèrement incliné et les pans latéraux ont été largement taillés en biseau pour mieux dégager l'espace. 


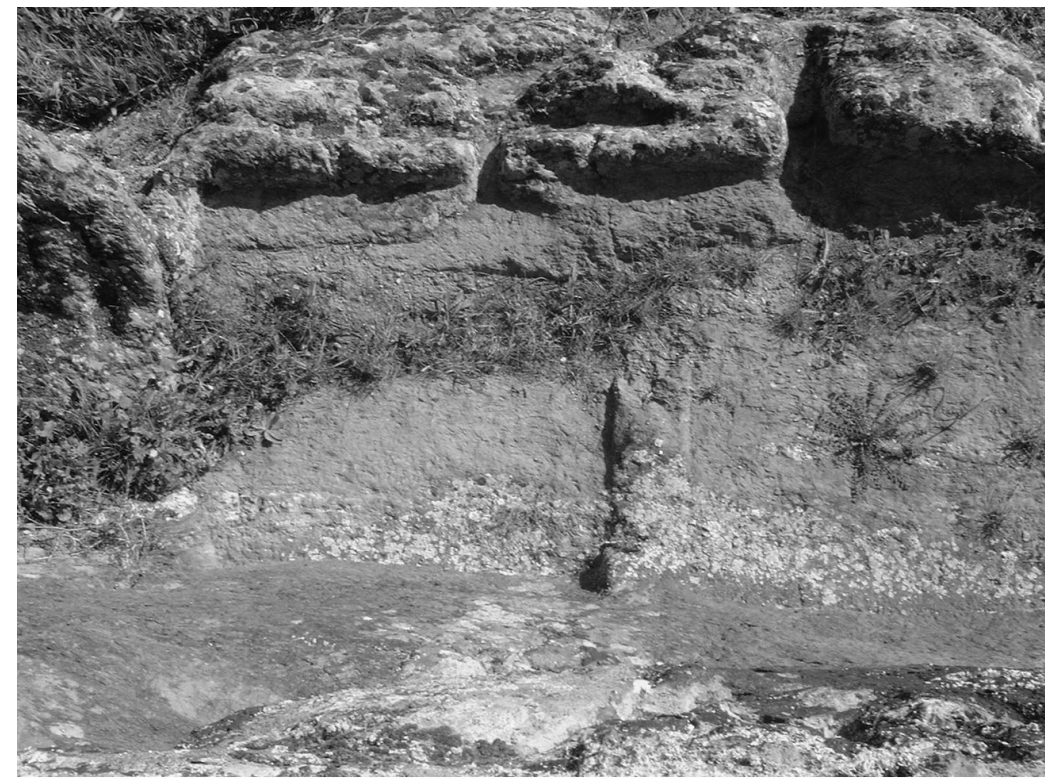

Рното 5. - Vue des bassins et de leur système d'évacuation depuis le sommet de la paroi principale. Le schiste à moins bien résisté à l'érosion du temps que ne l'aurait fait le granit, mais le soin apporté au travail de l'installation est encore nettement visible. La faible profondeur des cuvettes pouvait aisément être compensée par l'ajout de cadres de bois, bloqués entre les parois de pierre.

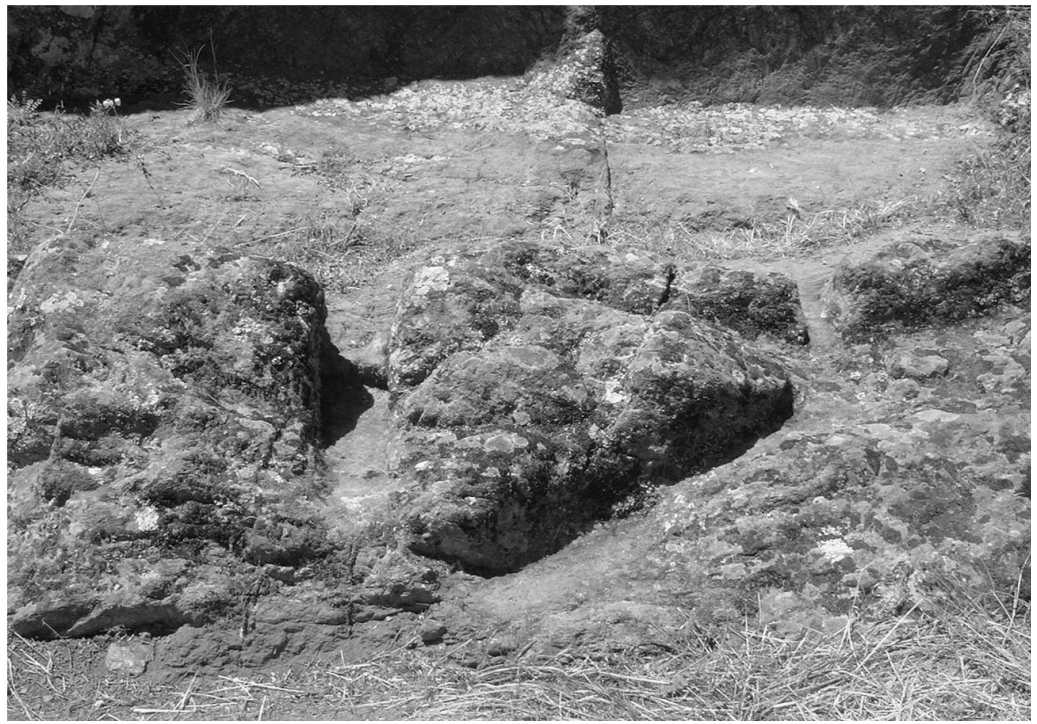

Рното 6. - Détail des évacuations. À gauche, l'écoulement depuis le fouloir s'effectue directement par une large goulotte surplombant une zone en dévers aujourd'hui en partie comblée. Elle recevait les récipients destinés à recueillir les jus exprimés. À droite, un canal venant du bassin de pressurage aboutissait au même endroit. 


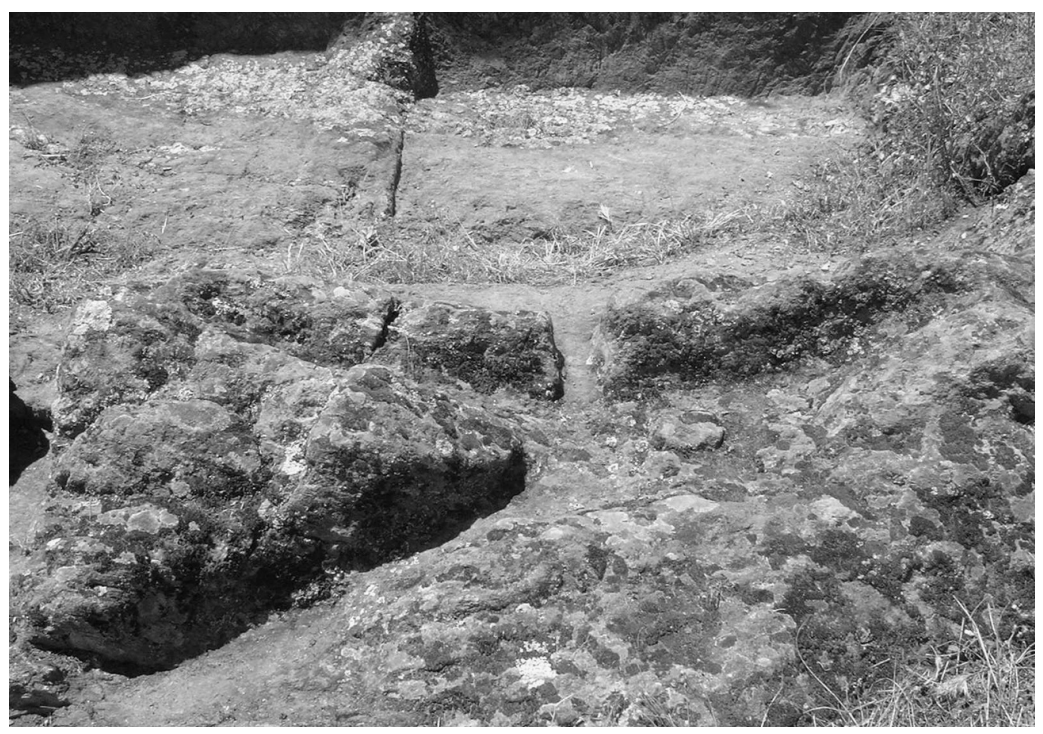

Рното 7. - Détail du système d'évacuation du bassin de pressurage. On aperçoit clairement en avant de la cuvette la goulotte en demie lune destinée à recueillir le trop plein appelé à se déverser par-dessus le muret frontal en cas de trop forte pression sur le marc. Le canal final d'évacuation, à la fois large et profond $(12 \mathrm{~cm})$, est semblable dans son profil à celui provenant du bassin de foulage par où également pouvait provenir une partie du jus en cas de débordement au niveau du muret de séparation.

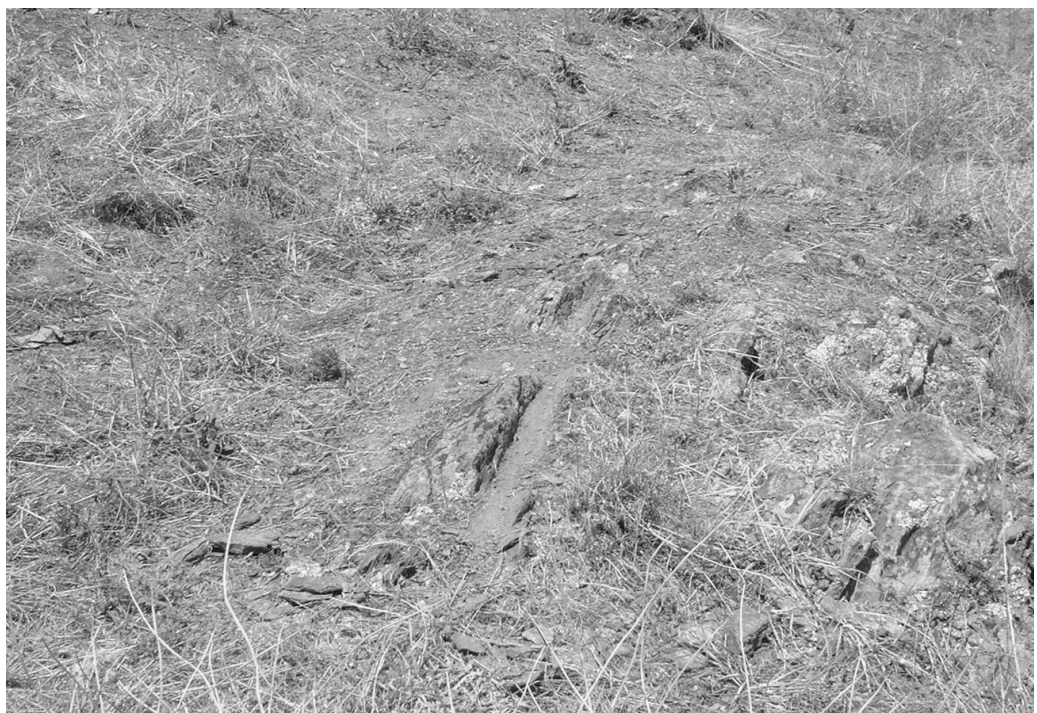

Рното 8. - Face au bassin de pressurage, à deux mètres de distance et légèrement en contrebas, le sol a été nivelé et la roche préparée pour recevoir les arbres de guidage du bras du pressoir. Dans la même ligne, mais un mètre plus loin et au niveau d'une terrasse inférieure (hors photographie), on relève l'emplacement probable du système de contrepoids. 